Organo- and

\title{
Chiral Tetraaminophosphonium Salts: A New Catalyst Motif
}

tetraaminophosphonium salts

Henry reaction

ion pairing

$$
\begin{aligned}
& \mathrm{R}^{1} \stackrel{\mathrm{O}}{\mathrm{H}}+\mathrm{R}^{2} \widehat{\mathrm{NO}_{2}} \\
& 2 \\
& 1 \\
& \mathrm{R}^{1}=\mathrm{Ar}, \text { Hetar, Alk, } \quad \mathrm{R}^{2}=\mathrm{H}, \mathrm{Me}, \mathrm{Et} \\
& \mathrm{PhCH}=\mathrm{CH}
\end{aligned}
$$

Proposed mechanism:

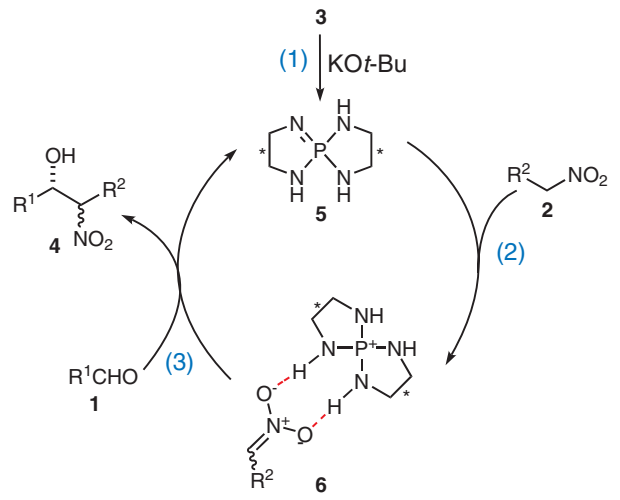

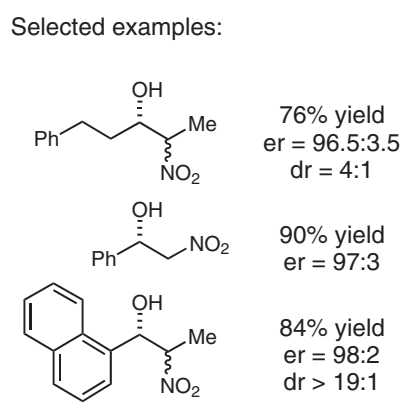

Significance: Ooi and co-workers have introduced chiral tetraaminophosphonium chlorides of type $\mathbf{3}$ as catalysts of the asymmetric Henry reaction of a broad range of aldehydes $\mathbf{1}$ and nitroalkanes $\mathbf{2}$. The authors' working hypothesis includes (1) the in situ generation of triaminoiminophosphorane $\mathbf{5}$ upon deprotonation of $\mathbf{3}$ with $\mathrm{KOt}$-Bu. (2) Phosphorane $\mathbf{5}$ will subsequently deprotonate nitroalkanes $\mathbf{2}$. The resulting chiral phosphonium nitronate ion pair $\mathbf{6}$ benefits from hydrogen bonding and enables (3) a highly stereoselective addition of aldehydes $\mathbf{1}$ in the final step. The ability of phosphonium salts $\mathbf{3}$ acting as hydrogen bonding donors via the $\mathrm{N}-\mathrm{H}$ protons could be demonstrated by an X-ray crystal structure of one representative $(\mathrm{Ar}=\mathrm{Ph})$.
Comment: The authors could show that tetraaminophosphonium salts besides serving as precursors of $\mathrm{P}-1$ phosphazenes exhibit truly catalytic potential. Access to phosphonium chlorides $\mathbf{3}$ is given by means of a concise ex-chiral-pool synthesis starting from L-valine. In the future, chiral P-spirocyclic salts of type $\mathbf{3}$ and related structures might also compete with the well-established $\mathrm{N}$-spirocyclic or cinchona alkaloid derived ammonium salts in phase-transfer catalysis. Ensuing reports on the use of chiral phosphonium salts in further reactions proceeding via anionic intermediates are expected. 